\title{
Entanglement in a QFT Model of Neutrino Oscillations
}

\author{
M. Blasone, ${ }^{1,2}$ F. Dell'Anno, ${ }^{2,3,4}$ S. De Siena, ${ }^{2,4,5}$ and F. Illuminati ${ }^{2,4,5}$ \\ ${ }^{1}$ Dipartimento di Fisica, Università degli Studi di Salerno, Via Giovanni Paolo II 132, 84084 Fisciano, Italy \\ ${ }^{2}$ INFN Sezione di Napoli, Gruppo Collegato di Salerno, Italy \\ ${ }^{3}$ Liceo Statale P.E. Imbriani, Via Pescatori 155, 83100 Avellino, Italy \\ ${ }^{4}$ Dipartimento di Ingegneria Industriale, Università degli Studi di Salerno, Via Giovanni Paolo II 132, 84084 Fisciano, Italy \\ ${ }^{5}$ Consorzio Nazionale Interuniversitario per le Scienze Fisiche della Materia (CNISM), Unità di Salerno, 84084 Fisciano, Italy \\ Correspondence should be addressed to M. Blasone; mblasone@unisa.it
}

Received 5 February 2014; Accepted 15 April 2014; Published 5 May 2014

Academic Editor: David Latimer

Copyright (C) 2014 M. Blasone et al. This is an open access article distributed under the Creative Commons Attribution License, which permits unrestricted use, distribution, and reproduction in any medium, provided the original work is properly cited. The publication of this article was funded by SCOAP S $^{3}$

\begin{abstract}
Tools of quantum information theory can be exploited to provide a convenient description of the phenomena of particle mixing and flavor oscillations in terms of entanglement, a fundamental quantum resource. We extend such a picture to the domain of quantum field theory where, due to the nontrivial nature of flavor neutrino states, the presence of antiparticles provides additional contributions to flavor entanglement. We use a suitable entanglement measure, the concurrence, that allows extracting the twomode (flavor) entanglement from the full multimode, multiparticle flavor neutrino states.
\end{abstract}

\section{Introduction}

In the last years, many efforts have been dedicated to the investigation of entanglement in the domain of elementary particle physics and quantum field theory [1-11]. The understanding of the role of nonlocal quantum correlations in infinite-dimensional systems of fields and particles, as well as the underlying mechanism governing their aggregation, represents a main goal. For systems composed by identical particles and/or sets of in general distinguishable field modes (either discrete or continuous, finite or infinite), the characterization and quantification of entanglement are achieved unambiguously only by properly taking into account the algebra of observables besides the tensor product structure of the individual state spaces. In the case of quantum fields, a further extension of such a framework is needed to take into account the correlations among distinguishable physical field modes rather than among indistinguishable particles and excitations [12-27].

For instance, the single-particle Bell superposition state $|0,1\rangle+|1,0\rangle$ between any two modes of the electromagnetic field is a well-known example of a maximally (bipartite) entangled quantum state (i.e., with maximal von Neumann entropy of the reduced single-mode density matrices), despite the fact that it involves only one excitation of the field (a single photon) [26]. In this case, the entanglement is between two different field modes with occupation numbers ranging between 0 and 1 . Considering single-particle or multiparticle (e.g., multiphoton) states of many modes leads to straightforward generalizations that allow considering the bipartite and multipartite multimode field entanglement of single-particle and multiparticle states. In the same way, it is possible to make precise sense of Bell nonlocality in the context of single-particle, multimode states [28-30].

In this context, it has been recently recognized that the phenomena of particle mixing and flavor oscillations can be understood in terms of quantum entanglement $[4,5]$. In particular, a connection has been established among the flavor transition probabilities and the multimode, single-particle entanglement for oscillating neutrinos [5]. Such a connection allows in principle engineering experimental protocols for the transfer of the quantum information encoded in neutrino states to spatially delocalized two-flavor charged lepton states $[4,5]$. The above analysis has been carried out in the context of quantum mechanics (QM), using the well-known Pontecorvo formalism for neutrino oscillations. On the other hand, it has been shown that flavor mixing in the context of quantum field theory (QFT) is associated with a highly 
nontrivial nature of the vacuum for the mixed fields [31-34]. As a consequence, neutrino states turn out to be multimode, multiparticle states, with a very rich structure of quantum correlations. In $[6,7]$, we studied the entanglement in such a system by means of entropic measures and found a relation with experimentally measurable quantities, like the variances of the lepton numbers and charges.

In the present work, we further investigate along this direction by adopting an alternative operational viewpoint on the entanglement associated with the system of oscillating neutrinos. Indeed, entanglement is an observable-induced, relative physical quantity [15-22], endowed with a specific operational meaning according to the selected reference quantum observables and quantum subsystems. By assuming the particle-antiparticle species as further quantum modes, we investigate the entanglement content of the neutrino system in the state obtained by tracing out the antiparticle species. Since such a state turns out to be a mixed one, we adopt the concurrence as a measure for the quantification of its entanglement content. Our results are in line with those of $[6,7]$ and naturally generalize the QM ones presented in [5].

The paper is organized as follows. In Section 2 we review the quantum information tools exploited in the paper and some results corresponding to the QM framework. In Section 3 we investigate the entanglement phenomenology of neutrino mixing and flavor oscillations adopting a QFT framework, and we discuss the nontrivial structure of flavor entanglement that emerges in the QFT framework.

\section{Entanglement and Flavor Oscillations: Quantum Mechanics}

In this section, we briefly review the background of the present analysis, that is, the formalism developed and the results obtained within the quantum mechanical framework $[4,5]$. Flavor mixing of neutrinos for two generations is described by the $2 \times 2$ rotation matrix $\mathbf{U}(\theta)$ [35]

$$
\mathbf{U}(\theta)=\left(\begin{array}{cc}
\cos \theta & \sin \theta \\
-\sin \theta & \cos \theta
\end{array}\right)
$$

where $\theta$ is the mixing angle. The two-flavor neutrino states are defined as

$$
\left|\underline{\nu}^{(f)}\right\rangle=\mathbf{U}(\theta)\left|\underline{\nu}^{(m)}\right\rangle
$$

where $\left|\underline{\nu}^{(f)}\right\rangle=\left(\left|\nu_{e}\right\rangle,\left|\nu_{\mu}\right\rangle\right)^{T}$ are the states with definite flavors $e, \mu$ and $\left|\underline{\nu}^{(m)}\right\rangle=\left(\left|v_{1}\right\rangle,\left|v_{2}\right\rangle\right)^{T}$ those with definite masses $m_{1}, m_{2}$. Both $\left|\nu_{\alpha}\right\rangle(\alpha=e, \mu)$ and $\left|v_{j}\right\rangle(j=1,2)$ are orthonormal. By describing the free propagation of the neutrino mass eigenstates with plane waves of the form $\left|\nu_{j}(t)\right\rangle=e^{-i \omega_{j} t}\left|\nu_{j}\right\rangle, \omega_{j}$ denoting the frequency associated with the mass eigenstate $\left|v_{j}\right\rangle$, the time evolution of the flavor states is given by

$$
\left|\underline{\nu}^{(f)}(t)\right\rangle=\mathbf{U}(t)\left|\underline{\nu}^{(f)}\right\rangle \equiv \mathbf{U}(\theta) \mathbf{U}_{0}(t) \mathbf{U}^{-1}(\theta)\left|\underline{\nu}^{(f)}\right\rangle,
$$

where $\left|\underline{v}^{(f)}\right\rangle$ are the flavor states at $t=0$, and $\mathbf{U}_{0}(t)=$ $\operatorname{diag}\left(e^{-i \omega_{1} t}, e^{-i \omega_{2} t}\right)$. By assuming the neutrino occupation number associated with a given flavor (mode) as reference quantum number, one can establish the following correspondences with two-qubit states:

$$
\begin{aligned}
& \left|v_{e}\right\rangle \equiv|1\rangle_{\nu_{e}}|0\rangle_{\nu_{\mu}} \equiv|10\rangle_{e \mu}, \\
& \left|v_{\mu}\right\rangle \equiv|0\rangle_{\nu_{e}}|1\rangle_{\nu_{\mu}} \equiv|01\rangle_{e \mu},
\end{aligned}
$$

where $|j\rangle_{v_{\alpha}}$ stands for a $j$-occupation number state of a neutrino in mode $\alpha$. Entanglement is thus established among flavor modes, in a single-particle setting. For instance, the free evolution of the electron-neutrino state $\left|\nu_{e}(t)\right\rangle$ can be written in the form

$$
\left|\nu_{e}(t)\right\rangle=\mathbf{U}_{e e}(t)|10\rangle_{e \mu}+\mathbf{U}_{e \mu}(t)|01\rangle_{e \mu},
$$

where $\left|\mathbf{U}_{e e}(t)\right|^{2}+\left|\mathbf{U}_{e \mu}(t)\right|^{2}=1$ due to normalization. Thus, the time-evolved states $\left|\underline{v}^{(f)}(t)\right\rangle$ are entangled Belllike superpositions of the two masses with time-independent coefficients or flavor eigenstates with time-dependent coefficients. It is worth remarking that the entanglement of (5) is in principle experimentally accessible, throughout a scheme for its transfer from single-neutrino states to two-flavor charged lepton states [5].

The entanglement content of the pure two-qubit state equation (5) is quantified by the von Neumann entropy of the reduced density matrix (or any other monotonic function of it), which is the widely accepted measure of the bipartite entanglement of pure states [36]. For other measures of entanglement (bipartite and multipartite systems), see [37$42]$. In the instance of mixed states, a particularly convenient measure for two-qubit systems is the concurrence $C(\rho)[43$, $44]$. For the particular case of a pair of qubits, such a measure is closely related to the entanglement of formation $E_{F}(\rho)$, which is the prototype of the convex-roof-based measures. The entanglement of formation has a simple physical interpretation: it is the minimal amount of entanglement needed for the production of a mixed state described by a given density matrix.

We briefly recall the definition of the concurrence, which will be used in this work. Let $\rho$ be the density operator corresponding to an arbitrary $N$-qubit state and describing a system partitioned into $N$ parties. The reduced density operator $\rho^{(\alpha ; \beta)}$ associated with $\rho$ is defined as

$$
\rho^{(\alpha ; \beta)}=\operatorname{Tr}_{\gamma \neq \alpha, \beta}[\rho] \text {, }
$$

where the trace operation is made over all the parties different from $\alpha$ and $\beta$. Next, the spin-flipped state $\tilde{\rho}^{(\alpha ; \beta)}$ reads

$$
\tilde{\rho}^{(\alpha ; \beta)}=\left(\sigma_{y} \otimes \sigma_{y}\right) \rho^{(\alpha ; \beta) *}\left(\sigma_{y} \otimes \sigma_{y}\right),
$$

where the complex conjugate is taken in the standard basis $\{|00\rangle,|01\rangle,|10\rangle,|11\rangle\}$. Then the concurrence is expressed in terms of square roots $\lambda_{i}$ of the eigenvalues of the nonHermitian matrix $\rho^{(\alpha ; \beta)} \tilde{\rho}^{(\alpha ; \beta)}$ :

$$
C\left(\rho^{(\alpha ; \beta)}\right)=\max \left\{0, \lambda_{1}-\lambda_{2}-\lambda_{3}-\lambda_{4}\right\},
$$




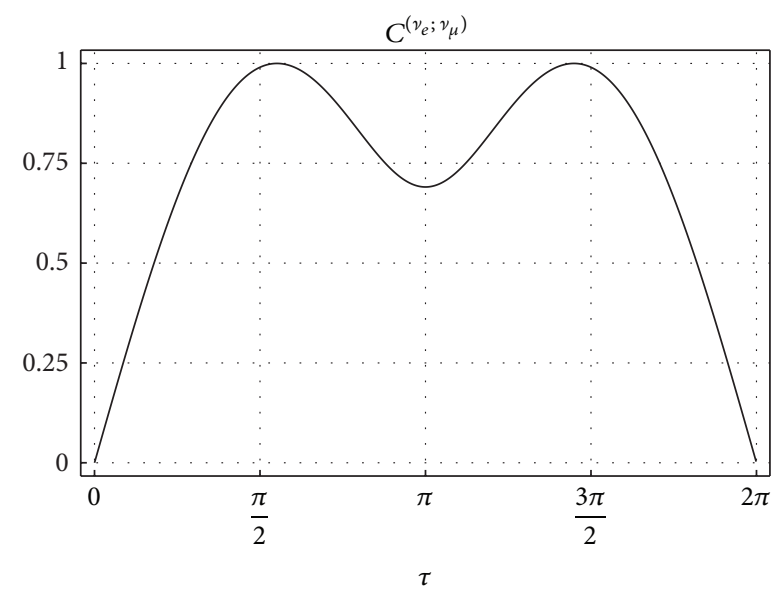

FIGURE 1: QM instance. The concurrence $C^{\left(\nu_{e} ; \nu_{\mu}\right)}$ as function of the scaled time $\tau=\left(\omega_{2}-\omega_{1}\right) t$. The mixing angle $\theta$ is fixed at the experimental value $\sin ^{2} \theta=0.314$.

where the $\lambda_{i}$ s are nonnegative real numbers taken in decreasing order with respect to the index $i$. The concurrence equation (8) can be easily computed for the pure two-qubit Bell state $\left|\nu_{e}(t)\right\rangle$, that is, (5), with density matrix $\rho_{e}=$ $\left|\nu_{e}(t)\right\rangle\left\langle v_{e}(t)\right|$. The concurrence $C^{\left(v_{e} ; \nu_{\mu}\right)} \equiv C\left(\rho^{\left(v_{e} \gamma_{\mu}\right)}\right)$ writes

$$
C^{\left(v_{e} ; v_{\mu}\right)}=2\left|\mathbf{U}_{e e}(t)\right|\left|\mathbf{U}_{e \mu}(t)\right|
$$

It is worth noticing that, in the instance of pure states, the concurrence coincides with the square root of the linear entropy. In Figure 1, we show the behavior of $C^{\left(\nu_{e} ; \nu_{\mu}\right)}$ as functions of the scaled, dimensionless time $\tau=\left(\omega_{2}-\omega_{1}\right) t$. At time $\tau=0$, the two flavors are not mixed, the entanglement is zero, and the global state of the system is factorized. For $\tau>0$, flavor oscillations occur, and the linear entropy exhibits a typical oscillatory behavior; the entanglement is maximal at largest mixing.

\section{Entanglement and Flavor Oscillations: Quantum Field Theory}

In this section, first we review the essential features of a specific QFT model of particle mixing describing the phenomena of neutrino oscillations [31,32]. For a general theory of mixing for an arbitrary number of fields see also [45].

Then, by using such a model, we present a generalization of the above analysis to the QFT framework. The neutrino Dirac fields $v_{e}(x)$ and $v_{\mu}(x)$ are defined through the mixing relations

$$
\begin{aligned}
& \nu_{e}(x)=\cos \theta v_{1}(x)+\sin \theta v_{2}(x), \\
& v_{\mu}(x)=-\sin \theta v_{1}(x)+\cos \theta v_{2}(x),
\end{aligned}
$$

where, in standard notation, $x$ stands for the four-vector $x \equiv$ $(t, \mathbf{x})$ and the free fields $\nu_{1}(x)$ and $\nu_{2}(x)$ with definite masses $m_{1}$ and $m_{2}$. The generator of the mixing transformations is given by

$$
G_{\theta}(t)=\exp \left[\theta \int d^{3} \mathbf{x}\left(v_{1}^{\dagger}(x) v_{2}(x)-v_{2}^{\dagger}(x) v_{1}(x)\right)\right]
$$

so that

$$
\nu_{\sigma}^{\alpha}(x)=G_{\theta}^{-1}(t) \nu_{i}^{\alpha}(x) G_{\theta}(t),
$$

where $(\sigma, i)=(e, 1),(\mu, 2)$, and the superscript $\alpha=1, \ldots, 4$ denotes the spinorial component. At finite volume, $G_{\theta}(t)$ is a unitary operator, that is, $G_{\theta}^{-1}(t)=G_{-\theta}(t)=G_{\theta}^{\dagger}(t)$, preserving the canonical anticommutation relations. The generator $G_{\theta}^{-1}(t)$ maps the Hilbert space for free fields $\mathscr{H}_{1,2}$ to the Hilbert space for mixed fields $\mathscr{H}_{e, \mu}$; that is, $G_{\theta}^{-1}(t)$ : $\mathscr{H}_{1,2} \mapsto \mathscr{H}_{e, \mu}$. In particular, the flavor vacuum is given by $|0(t)\rangle_{e, \mu}=G_{\theta}^{-1}(t) \quad|0\rangle_{1,2} \quad$ at finite volume $V$. We denote by $|0\rangle_{e, \mu}$ the flavor vacuum at $t=0$. It is worth noticing that, in the infinite volume limit, the flavor and the mass vacua are unitarily inequivalent. The free fields $v_{i}(x)(i=1,2)$ are given by the following expansions:

$$
v_{i}(x)=\frac{1}{\sqrt{V}} \sum_{\mathbf{k}, r}\left[u_{\mathbf{k}, i}^{r} \alpha_{\mathbf{k}, i}^{r}(t)+v_{-\mathbf{k}, i}^{r} \beta_{-\mathbf{k}, i}^{r \dagger}(t)\right] e^{i \mathbf{k} \cdot \mathbf{x}},
$$

where $\mathbf{k}$ is the momentum vector, $r=1,2$ denotes the helicity, $\alpha_{\mathbf{k}, i}^{r}(t)=\alpha_{\mathbf{k}, i}^{r} e^{-i \omega_{k, i} t}, \beta_{\mathbf{k}, i}^{r \dagger}(t)=\beta_{\mathbf{k}, i}^{r \dagger} e^{i \omega_{k, i} t}$, and $\omega_{k, i}=$ $\sqrt{\mathbf{k}^{2}+m_{i}^{2}}$. The operators $\alpha_{\mathbf{k}, i}^{r}$ and $\beta_{\mathbf{k}, i}^{r}$ are the annihilation operators for the vacuum state $|0\rangle_{m} \equiv|0\rangle_{1} \otimes|0\rangle_{2}$; that is, $\alpha_{\mathbf{k}, i}^{r}|0\rangle_{m}=\beta_{\mathbf{k}, i}^{r}|0\rangle_{m}=0$. The anticommutation relations are the usual ones; extended details, for example, on the orthonormality and completeness relations are presented in our previous works $[31,32]$. By use of $G_{\theta}(t)$, the flavor fields can be expanded as

$$
v_{\sigma}(\mathbf{x})=\frac{1}{\sqrt{V}} \sum_{\mathbf{k}, r}\left[u_{\mathbf{k}, i}^{r} \alpha_{\mathbf{k}, \sigma}^{r}(t)+v_{-\mathbf{k}, i}^{r} \beta_{-\mathbf{k}, \sigma}^{r \dagger}(t)\right] e^{i \mathbf{k} \cdot \mathbf{x}} .
$$

The flavor annihilation operators are defined as $\alpha_{\mathbf{k}, \sigma}^{r}(t) \equiv$ $G_{\theta}^{-1}(t) \alpha_{\mathbf{k}, i}^{r} G_{\theta}(t)$ and $\beta_{\mathbf{k}, \sigma}^{r \dagger}(t) \equiv G_{\theta}^{-1}(t) \beta_{\mathbf{k}, i}^{r \dagger} G_{\theta}(t)$. Without any loss of generality, let us choose the reference frame such that $\mathbf{k}=(0,0,|\mathbf{k}|)$; we have

$$
\begin{aligned}
\alpha_{\mathbf{k}, e}^{r}(t)= & \cos \theta \alpha_{\mathbf{k}, 1}^{r}(t) \\
& +\sin \theta\left(\left|U_{\mathbf{k}}\right| \alpha_{\mathbf{k}, 2}^{r}(t)+\epsilon^{r}\left|V_{\mathbf{k}}\right| \beta_{-\mathbf{k}, 2}^{r \dagger}(t)\right),
\end{aligned}
$$

where $\epsilon^{r}=(-1)^{r}$ and

$$
\begin{aligned}
\left|U_{\mathbf{k}}\right| & \equiv u_{\mathbf{k}, i}^{r \dagger} u_{\mathbf{k}, j}^{r}=v_{-\mathbf{k}, i}^{r \dagger} v_{-\mathbf{k}, j}^{r} \\
& =\frac{|\mathbf{k}|^{2}+\left(\omega_{k, 1}+m_{1}\right)\left(\omega_{k, 2}+m_{2}\right)}{2 \sqrt{\omega_{k, 1} \omega_{k, 2}\left(\omega_{k, 1}+m_{1}\right)\left(\omega_{k, 2}+m_{2}\right)}}, \\
\left|V_{\mathbf{k}}\right| & \equiv \epsilon^{r} u_{\mathbf{k}, 1}^{r \dagger} v_{-\mathbf{k}, 2}^{r}=-\epsilon^{r} u_{\mathbf{k}, 2}^{r \dagger} v_{-\mathbf{k}, 1}^{r} \\
& =\frac{\left(\omega_{k, 1}+m_{1}\right)-\left(\omega_{k, 2}+m_{2}\right)}{2 \sqrt{\omega_{k, 1} \omega_{k, 2}\left(\omega_{k, 1}+m_{1}\right)\left(\omega_{k, 2}+m_{2}\right)}}|\mathbf{k}|,
\end{aligned}
$$


with $i, j=1,2, i \neq j$, and $\left|U_{\mathbf{k}}\right|^{2}+\left|V_{\mathbf{k}}\right|^{2}=1$. The explicit expression for the flavor states $\left|\nu_{\mathbf{k}, e}^{r}\right\rangle$ at time $t=0$ is

$$
\begin{aligned}
\left|\nu_{\mathbf{k}, e}^{r}\right\rangle \equiv & \alpha_{\mathbf{k}, e}^{r \dagger}(0)|0\rangle_{e, \mu} \\
= & {\left[\cos \theta \alpha_{\mathbf{k}, 1}^{r \dagger}+\left|U_{\mathbf{k}}\right| \sin \theta \alpha_{\mathbf{k}, 2}^{r \dagger}\right.} \\
& \left.\quad-\epsilon^{r}\left|V_{\mathbf{k}}\right| \sin \theta \alpha_{\mathbf{k}, 1}^{r \dagger} \alpha_{\mathbf{k}, 2}^{r \dagger} \beta_{-\mathbf{k}, 1}^{r \dagger}\right] \\
& \times G_{\theta, \mathbf{k}, r}^{-1}(t) \prod_{\mathbf{p} \neq \mathbf{k}} G_{\theta, \mathbf{p}, s}^{-1}(t)|0\rangle_{1,2},
\end{aligned}
$$

where $G_{\theta}(t)=\prod_{\mathbf{p}} \prod_{s=1}^{2} G_{\theta, \mathbf{p}, s}(t)$. In the state (17), a multiparticle component is present, disappearing in the relativistic limit $|\mathbf{k}| \gg \sqrt{m_{1} m_{2}}$ : indeed, for large $|\mathbf{k}|$, since one gets $\left|U_{\mathbf{k}}\right|^{2} \rightarrow 1$ and $\left|V_{\mathbf{k}}\right|^{2} \rightarrow 0$, the (quantum-mechanical) Pontecorvo states are recovered. In order to simplify the notation, we omit the superscript $r$ (by fixing $r=2$ ) and the subscript $\mathbf{k}$, thus restricting the analysis to the flavor neutrino state $\left|v_{e}\right\rangle$ of fixed momentum and helicity. Let us consider again the free evolution of the electron-neutrino state (17):

$$
\left|\nu_{e}(t)\right\rangle=e^{-i H_{0} t}\left|\nu_{e}\right\rangle,
$$

where $H_{0}$ is the standard QFT free Hamiltonian. Finally, in the Hilbert space $\mathscr{H}_{e, \mu},(18)$ can be written in the form

$$
\begin{aligned}
\left|\nu_{e}(t)\right\rangle=[ & \mathbf{U}_{e e}(t) \alpha_{e}^{\dagger}+\mathbf{U}_{e \mu}(t) \alpha_{\mu}^{\dagger}+\mathbf{U}_{e \mu}^{e \bar{e}}(t) \alpha_{e}^{\dagger} \alpha_{\mu}^{\dagger} \beta_{e}^{\dagger} \\
& \left.+\mathbf{U}_{e e}^{\mu \bar{\mu}}(t) \alpha_{e}^{\dagger} \alpha_{\mu}^{\dagger} \beta_{\mu}^{\dagger}\right]|0\rangle_{e, \mu},
\end{aligned}
$$

where the time-dependent coefficients are given by

$$
\begin{aligned}
& \mathbf{U}_{e e}(t)=e^{-i \omega_{1} t}\left[\cos ^{2} \theta+\sin ^{2} \theta\left(e^{-i\left(\omega_{2}-\omega_{1}\right) t}|U|^{2}\right.\right. \\
& \left.\left.+e^{-i\left(\omega_{2}+\omega_{1}\right) t}|V|^{2}\right)\right], \\
& \mathbf{U}_{e \mu}(t)=e^{-i \omega_{1} t} U \cos \theta \sin \theta\left(e^{-i\left(\omega_{2}-\omega_{1}\right) t}-1\right), \\
& \mathbf{U}_{e \mu}^{e \bar{e}}(t)=e^{-i \omega_{1} t} V \cos \theta \sin \theta\left(1-e^{-i\left(\omega_{2}+\omega_{1}\right) t}\right), \\
& \mathbf{U}_{e e}^{\mu \bar{\mu}}(t)=e^{-i \omega_{1} t} U V \sin ^{2} \theta\left(e^{-i\left(\omega_{2}+\omega_{1}\right) t}-e^{-i\left(\omega_{2}-\omega_{1}\right) t}\right), \\
& \left|\mathbf{U}_{e e}(t)\right|^{2}+\left|\mathbf{U}_{e \mu}(t)\right|^{2}+\left|\mathbf{U}_{e \mu}^{e \bar{e}}(t)\right|^{2}+\left|\mathbf{U}_{e e}^{\mu \bar{\mu}}(t)\right|^{2}=1 .
\end{aligned}
$$

In the following, in order to conveniently parameterize the neutrino masses $m_{1}$ and $m_{2}$, momentum $|\mathbf{k}|$, and the evolution time $t$, we use the real parameters $x=m_{2} / m_{1}$, $p=|\mathbf{k}| / \sqrt{m_{1} m_{2}}$, and $\tau=\left(\omega_{2}-\omega_{1}\right) t$. Therefore, $x$ represents the ratio between the two masses eigenvalues; $p$ expresses the ratio between the momentum and the masses geometrical mean and corresponds to the relativistic limit for $p \gg 1$.

Evidently, the time-evolved state $\left|v_{e}(t)\right\rangle$ in the flavor eigenstates Hilbert space, that is, (19), is a multiparticle entangled state. Analogously with the Pontecorvo states (5), we assume the neutrino occupation number as reference quantum number. However, with respect to (5), we have still two flavors, but we have a further degree of freedom, that

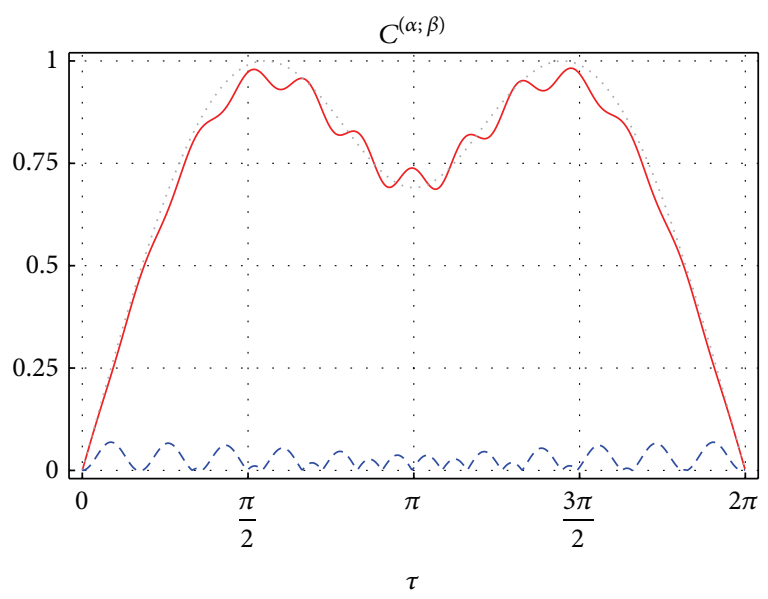

FIGURE 2: QFT instance (color online). The concurrences $C^{\left(\nu_{e} ; \nu_{\mu}\right)}$ (full line) and $C^{\left(\bar{\nu}_{e} ; \bar{\nu}_{\mu}\right)}$ (dashed line) as functions of the scaled time $\tau=\left(\omega_{2}-\omega_{1}\right) t$. The mixing angle $\theta$ is fixed at the experimental value $\sin ^{2} \theta=0.314$; the parameters $x$ and $p$ are fixed as $x=10$ and $p=5$. The concurrence associated with the quantum-mechanical Pontecorvo states (dotted line), that is, Figure 1, is also reported for comparison.

is, the neutrino species, that is, particles and antiparticles. Therefore, we obtain multipartite entanglement in a fourqubit state. In the instance $\mathscr{H}_{e, \mu}$, (19) can be written in the form

$$
\begin{aligned}
\left|\nu_{e}(t)\right\rangle= & \mathbf{U}_{e e}(t)|1000\rangle_{e \mu \bar{e} \bar{\mu}}+\mathbf{U}_{e \mu}(t)|0100\rangle_{e \mu \bar{e} \bar{\mu}} \\
& +\mathbf{U}_{e \mu}^{e \bar{e}}(t)|1110\rangle_{e \mu \bar{\mu} \bar{\mu}}+\mathbf{U}_{e e}^{\mu \bar{\mu}}(t)|1101\rangle_{e \mu \bar{e} \bar{\mu}}
\end{aligned}
$$

where $|i j k h\rangle_{e \mu \bar{e} \bar{\mu}}$ denotes the four-qubit vector $|i\rangle_{\nu_{e}}|j\rangle_{\nu_{\mu}}|k\rangle_{\bar{v}_{e}}|h\rangle_{\bar{\nu}_{\mu}}$ with $i, j, k, h=0,1$. Let us analyze the entanglement content possessed by a given pair of modes of $\left|v_{e}\right\rangle$, that is, (21), by using the concurrence, that is, by (8). Specifically, we compute the quantity $C^{(\alpha ; \beta)}$ associated with the two modes $\alpha \neq \beta=v_{e}, v_{\mu}, \bar{v}_{e}, \bar{v}_{\mu}$. We do not report the analytical expressions for the concurrences, as they are long and unwieldy and provide no further significant physical insight.

In Figure 2, we plot the quantities $C^{\left(v_{e} ; \nu_{\mu}\right)}$ and $C^{\left(\bar{\nu}_{e} ; \bar{\nu}_{\mu}\right)}$ as functions of the scaled time $\tau$ for $x=10$ and $p=5$; it is worth noticing that such a choice of the parameters corresponds to the following assumptions: mass $m_{2}$ greater than mass $m_{1}$ of one order of magnitude and momentum of the same order of magnitude as the masses geometrical mean. We observe that the particle-mode entanglement $\left(v_{e} ; v_{\mu}\right)$ is predominant; thus, most entanglement is shared between the two particle modes. Notwithstanding, a nonvanishing (although suppressed), nontrivial, oscillating contribution originates from the antiparticle-mode pair $\left(\bar{v}_{e}, \bar{v}_{\mu}\right)$.

For completeness, in Figure 3 we also plot the concurrences $C^{\left(\nu_{e} \cdot \bar{\nu}_{e}\right)}$ and $C^{\left(\nu_{\mu} ; \bar{\nu}_{e}\right)}$, panel (a), and $C^{\left(\nu_{e} ; \bar{\nu}_{\mu}\right)}$ and $C^{\left(\nu_{\mu} ; \bar{\nu}_{\mu}\right)}$, panel (b). Specifically, plots in Figure 3 represent the concurrences associated with the pairs particle-antiparticle $\left(\nu_{\alpha} ; \bar{v}_{\beta}\right)$. We observe that there exists strong entanglement content in these pairs; this fact is due to the Bogoliubov contribution in (15). Let us notice that the curves $C^{\left(v_{e} ; \bar{\nu}_{\beta}\right)}$ and $C^{\left(\nu_{\mu} \bar{\nu}_{\beta}\right)}$ (with 


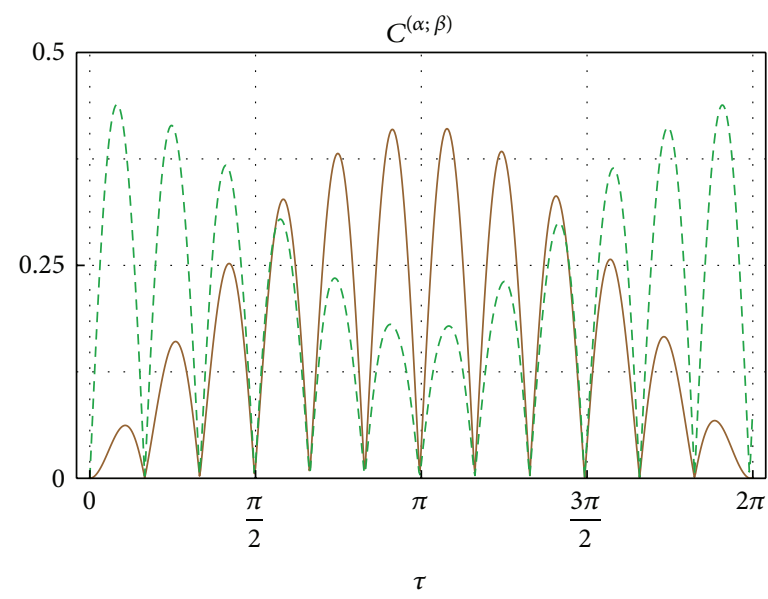

(a)

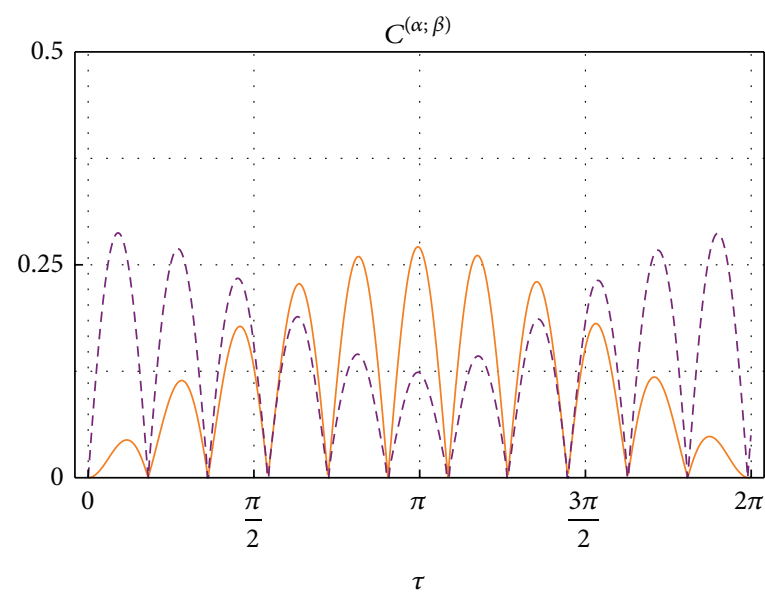

(b)

FIGURE 3: QFT instance (color online). In (a) the concurrences $C^{\left(v_{e} ; \bar{v}_{e}\right)}$ (full line) and $C^{\left(\nu_{\mu} i \bar{v}_{e}\right)}$ (dashed line), in (b) the concurrences $C^{\left(v_{e} ; \bar{\nu}_{\mu}\right)}$ (full line) and $C^{\left(\nu_{\mu}, \bar{\nu}_{\mu}\right)}$ (dashed line), as functions of the scaled time $\tau=\left(\omega_{2}-\omega_{1}\right) t$. The mixing angle $\theta$ and the parameters $x$ and $p$ are fixed as in Figure 2.

$\beta=e, \mu)$ exhibit an opposite behavior; on average, when the former increases (decreases), the latter decreases (increases).

\section{Conclusions}

In this paper, we analyzed a paradigmatic phenomenon of particle physics, that is, neutrino oscillations, from the point of view of entanglement, one of the fundamental aspects of quantum theory. More specifically, we studied the entanglement associated with a QFT model of neutrino oscillations, generalizing our previous results derived in the context of QM. The two-mode state, obtained by tracing out two modes, is a mixed one, and we characterized the entanglement of such a state by means of the concurrence.

We showed that such a phenomenon, described in a QFT framework, exhibits significantly more complex effects with respect to that found in a QM setting. This procedure is applicable as well to other multiparticle QFT systems, beyond the model considered here.

The present analysis has been carried out for the case of two generations. A further extension can be carried out by considering the case of three flavors, including CP violation, for which the structure of QFT flavor states is considerably more involved [34].

Moreover, the entanglement dynamics has been studied in the time domain; an analogous study can be carried out in the space domain, by considering the spatial distribution of entanglement.

At last, it is also worth remarking that the (exact) results obtained in our paper represent a canonical example of evaluation of entanglement for a relativistic system.

\section{Conflict of Interests}

The authors declare that there is no conflict of interests regarding the publication of this paper.

\section{Acknowledgment}

The authors acknowledge support from the EU STREP Project iQIT, Grant no. 270843.

\section{References}

[1] R. A. Bertlmann and W. Grimus, "Quantum-mechanical interference over macroscopic distances in the $B^{0} \bar{B}^{0}$ system," Physics Letters B, vol. 392, no. 3-4, pp. 426-432, 1997.

[2] R. A. Bertlmann, W. Grimus, and B. C. Hiesmayr, "Bell inequality and CP violation in the neutral kaon system," Physics Letters A, vol. 289, no. 1-2, pp. 21-26, 2001.

[3] A. Di Domenico, A. Gabriel, B. C. Hiesmayr et al., "Heisenberg's uncertainty relation and bell inequalities in high energy physics: an effective formalism for unstable two-state systems," Foundations of Physics, vol. 42, no. 6, pp. 778-802, 2012.

[4] M. Blasone, F. Dell'Anno, S. De Siena, M. Di Mauro, and F. Illuminati, "Multipartite entangled states in particle mixing," Physical Review D, vol. 77, Article ID 096002, 2008.

[5] M. Blasone, F. Dell'Anno, S. De Siena, and F. Illuminati, "Entanglement in neutrino oscillations," Europhysics Letters, vol. 85, no. 5, Article ID 50002, 2009.

[6] M. Blasone, F. Dell'Anno, S. De Siena, and F. Illuminati, "Entanglement in quantum field theory: particle mixing and oscillations," Journal of Physics: Conference Series, vol. 442, no. 1, Article ID 012070, 2013.

[7] M. Blasone, F. Dell'Anno, S. De Siena, and F. Illuminati, "A fieldtheoretical approach to entanglement in neutrino mixing and oscillations," In press, http://arxiv.org/abs/1401.7793.

[8] B. Kayser, J. Kopp, R. G. H. Robertson, and P. Vogel, “Theory of neutrino oscillations with entanglement," Physical Review D, vol. 82, Article ID 093003, 2010.

[9] E. K. Akhmedov and A. Y. Smirnov, "Neutrino oscillations: entanglement, energy-momentum conservation and QFT," Foundations of Physics, vol. 41, no. 8, pp. 1279-1306, 2011. 
[10] D. Boyanovsky, "Short baseline neutrino oscillations: when entanglement suppresses coherence," Physical Review D, vol. 84, Article ID 065001, 2011.

[11] L. Lello, D. Boyanovsky, and R. Holman, "Entanglement entropy in particle decay," Journal of High Energy Physics, vol. 2013, article 116, 2013.

[12] G. Ghirardi and L. Marinatto, "Criteria for the entanglement of composite systems with identical particles," Fortschritte der Physik, vol. 52, no. 11-12, pp. 1045-1051, 2004.

[13] F. Benatti, R. Floreanini, and U. Marzolino, "Bipartite entanglement in systems of identical particles: the partial transposition criterion," Annals of Physics, vol. 327, no. 5, pp. 1304-1319, 2012.

[14] F. Benatti, R. Floreanini, and U. Marzolino, "Sub-shot-noise quantum metrology with entangled identical particles," Annals of Physics, vol. 325, no. 4, pp. 924-935, 2010.

[15] P. Zanardi, "Quantum entanglement in fermionic lattices," Physical Review A, vol. 65, Article ID 042101, 2002.

[16] P. Zanardi and X. Wang, "Fermionic entanglement in itinerant systems," Journal of Physics A: Mathematical and General, vol. 35, no. 37, article 7947, 2002.

[17] P. Zanardi, "Bipartite mode entanglement of bosonic condensates on tunneling graphs," Physical Review A, vol. 67, Article ID 054301, 2003.

[18] P. Giorda and P. Zanardi, "Mode entanglement and entangling power in bosonic graphs," Physical Review A, vol. 68, Article ID 062108, 2003.

[19] E. Ciancio, P. Giorda, and P. Zanardi, "Mode transformations and entanglement relativity in bipartite Gaussian states," Physics Letters A, vol. 354, no. 4, pp. 274-280, 2006.

[20] P. Zanardi, D. A. Lidar, and S. Lloyd, "Quantum tensor product structures are observable induced," Physical Review Letters, vol. 92, Article ID 060402, 2004.

[21] H. M. Wiseman and J. A. Vaccaro, "Entanglement of indistinguishable particles shared between two parties," Physical Review Letters, vol. 91, Article ID 097902, 2003.

[22] M. R. Dowling, A. C. Doherty, and H. M. Wiseman, "Entanglement of indistinguishable particles in condensed-matter physics," Physical Review A, vol. 79, Article ID 052323, 2006.

[23] H. Barnum, E. Knill, G. Ortiz, R. Somma, and L. Viola, "A subsystem-independent generalization of entanglement," Physical Review Letters, vol. 92, Article ID 107902, 2004.

[24] H. Barnum, G. Ortiz, R. Somma, and L. Viola, "A generalization of entanglement to convex operational theories: entanglement relative to a subspace of observables," International Journal of Theoretical Physics, vol. 44, no. 12, pp. 2127-2145, 2005.

[25] M.-C. Banuls, J. I. Cirac, and M. M. Wolf, "Entanglement in fermionic systems," Physical Review A, vol. 76, Article ID 022311, 2006.

[26] S. J. van Enk, "Single-particle entanglement," Physical Review A, vol. 72, Article ID 064306, 2005.

[27] M. O. T. Cunha, J. A. Dunningham, and V. Vedral, "Entanglement in single-particle systems," Proceedings of the Royal Society A: Mathematical, Physical and Engineering Sciences, vol. 463, no. 2085, pp. 2277-2286, 2007.

[28] J. Dunningham and V. Vedral, "Nonlocality of a single particle," Physical Review Letters, vol. 99, Article ID 180404, 2007.

[29] J. Dunningham, V. Palge, and V. Vedral, "Entanglement and nonlocality of a single relativistic particle," Physical Review A, vol. 80, Article ID 044302, 2009.
[30] L. Heaney, A. Cabello, M. F. Santos, and V. Vedral, "Extreme nonlocality with one photon," New Journal of Physics, vol. 13, Article ID 053054, 2011.

[31] M. Blasone and G. Vitiello, "Quantum field theory of fermion mixing," Annals of Physics, vol. 244, no. 2, pp. 283-311, 1995.

[32] M. Blasone, P. A. Henning, and G. Vitiello, "The exact formula for neutrino oscillations," Physics Letters B: Nuclear, Elementary Particle and High-Energy Physics, vol. 451, no. 1-2, pp. 140-145, 1999.

[33] M. Blasone, P. Jizba, and G. Vitiello, "Currents and charges for mixed fields," Physics Letters B: Nuclear, Elementary Particle and High-Energy Physics, vol. 517, no. 3-4, pp. 471-475, 2001.

[34] M. Blasone, A. Capolupo, and G. Vitiello, "Quantum field theory of three flavor neutrino mixing and oscillations with CP violation," Physical Review D, vol. 66, no. 2, Article ID 025033, 2002.

[35] C. Giunti and C. W. Kim, Fundamentals of Neutrino Physics and Astrophysics, Oxford University Press, Oxford, UK, 2007.

[36] R. Horodecki, P. Horodecki, M. Horodecki, and K. Horodecki, "Quantum entanglement," Reviews of Modern Physics, vol. 81, no. 2, pp. 865-942, 2009.

[37] H. Barnum and N. Linden, "Monotones and invariants for multi-particle quantum states," Journal of Physics A: Mathematical and General, vol. 34, no. 35, pp. 6787-6805, 2001.

[38] D. A. Meyer and N. R. Wallach, "Global entanglement in multiparticle systems," Journal of Mathematical Physics, vol. 43, no. 9, pp. 4273-4278, 2002.

[39] G. K. Brennen, "An observable measure of entanglement for pure states of multi-qubit systems," Quantum Information \& Computation, vol. 3, no. 6, pp. 619-626, 2003.

[40] T.-C. Wei and P. M. Goldbart, "Geometric measure of entanglement and applications to bipartite and multipartite quantum states," Physical Review A, vol. 68, Article ID 042307, 2003.

[41] T. R. de Oliveira, G. Rigolin, and M. C. de Oliveira, "Genuine multipartite entanglement in quantum phase transitions," Physical Review A, vol. 73, Article ID 010305(R), 2006.

[42] M. Blasone, F. Dell'Anno, S. de Siena, and F. Illuminati, "Hierarchies of geometric entanglement," Physical Review A, vol. 77, no. 6, Article ID 062304, 2008.

[43] W. K. Wootters, "Entanglement of formation of an arbitrary state of two qubits," Physical Review Letters, vol. 80, no. 10, pp. 2245-2248, 1998.

[44] S. Hill and W. K. Wootters, "Entanglement of a pair of quantum bits," Physical Review Letters, vol. 78, no. 26, pp. 5022-5025, 1997.

[45] C. -R. Ji and Y. Mishchenko, "General theory of quantum field mixing," Physical Review D, vol. 65, Article ID 096015, 2002. 

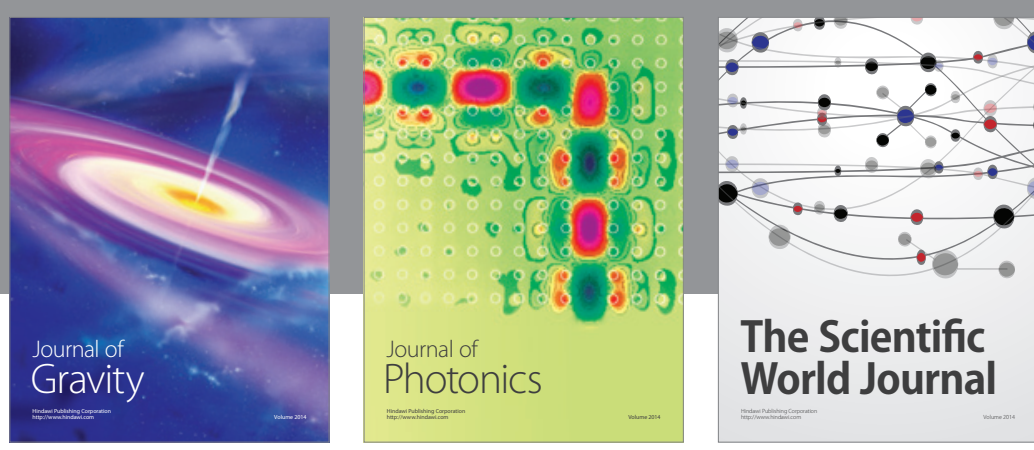

The Scientific World Journal
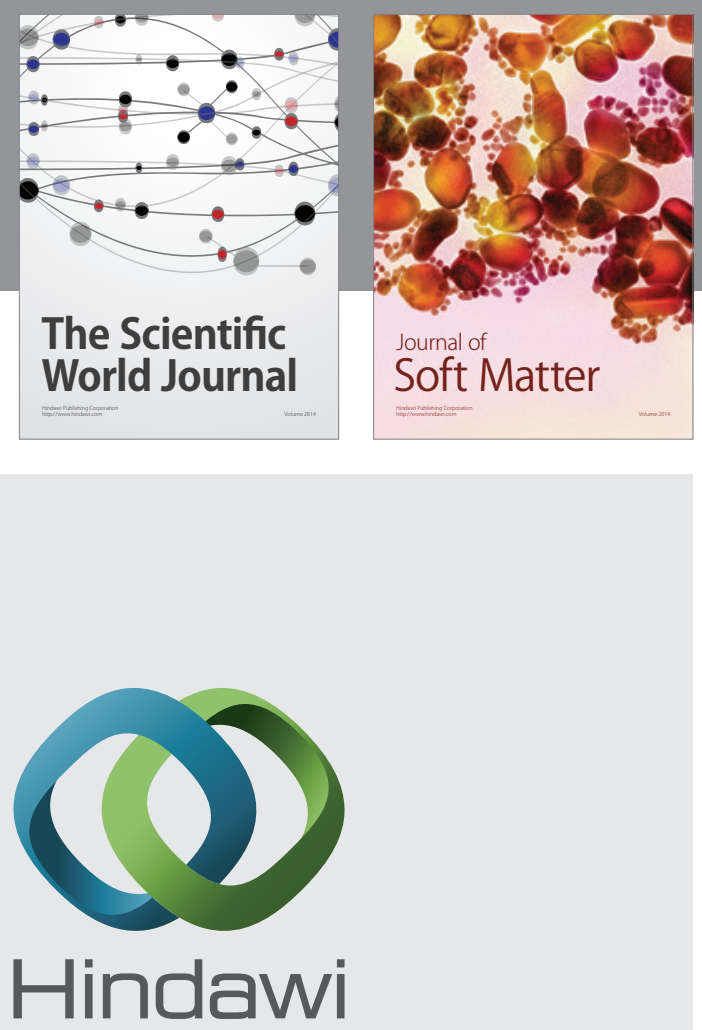

Submit your manuscripts at

http://www.hindawi.com

nternational Journal of

Statistical Mechanics
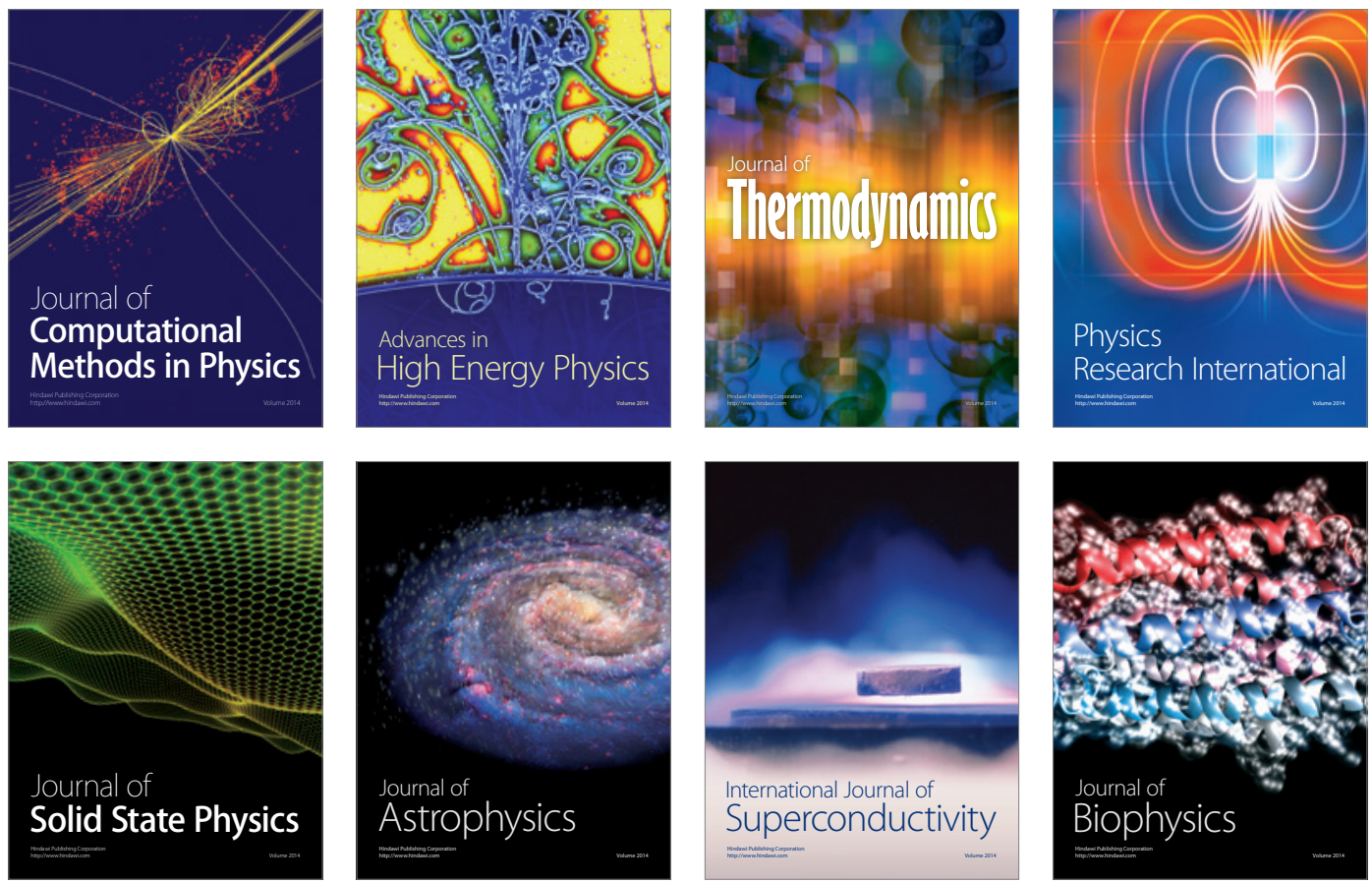
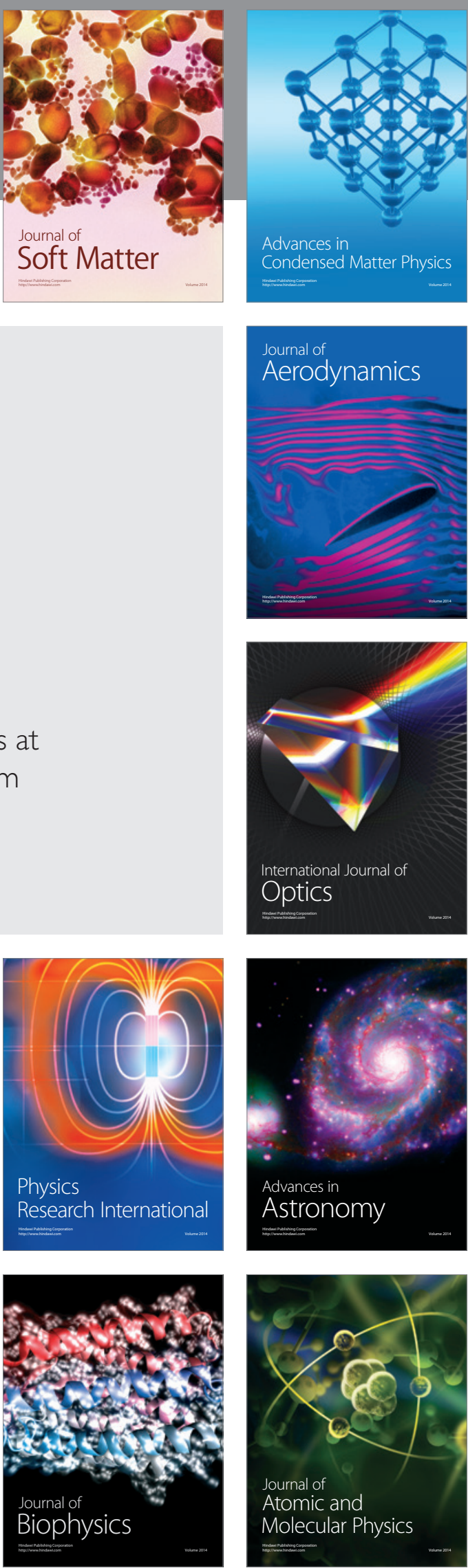